\title{
Performance evaluation of high-lift hydrofoils with a flap used in the design of horizontal-axis hydrokinetic turbines
}

\author{
Rubio-Clemente $\mathrm{A}^{\mathbf{1 , 2}}$, Aguilar $\mathrm{J}^{2}$, Chica $\mathrm{E}^{2}$ \\ ${ }^{1}$ Facultad de Ingeniería, Universidad de Medellín, Carrera 87, No. 30-65, Medellín, Colombia. \\ ${ }^{2}$ Grupo de Investigación Energía Alternativa, Facultad de Ingeniería, Universidad de Antioquia UdeA, Calle 70, No. 52-21, \\ Medellín, Colombia. \\ Phone/Fax number: 0057(4)3405555, e-mail: arubio@udem.edu.co
}

\begin{abstract}
The hydrodynamic performance and the flow field of two horizontal-axis hydrokinetic turbines with and without a high-lift hydrofoil with a flap were investigated using computational fluid dynamics (CFD) simulation. For improving the accuracy of the numerical simulation, the user-defined function (UDF) of 6-degrees of freedom (6-DoF) was used in the Ansys Fluent software. Unsteady Reynolds-averaged Navier-Stokes (URANS) equations coupled to the SST $k-\omega$ turbulence model were employed during the simulation. A three-dimensional model of both of the turbines with three blades was conducted for obtaining the performance curve of the power coefficient $\left(C_{P}\right)$ versus the tip speed ratio (TSR). The maximum power coefficients $\left(C_{P_{\text {Max }}}\right)$ of the hydrokinetic turbines with and without a high-lift hydrofoil arrangement were 0.5050 and 0.419 , respectively. Experimental data from the literature were used for the validation of the numerical results, specifically for the case when a rotor with traditional blades is utilized. In general, the simulation results were in good agreement with the experimental data.
\end{abstract}

Key words. Hydrofoil-flap arrangement, 6-DoF, hydrokinetic turbine, high-lift hydrofoil, Richardson extrapolation.

\section{Introduction}

Hydrokinetic turbines should be optimally designed for transforming the kinetic energy contained in river, tidal and marine currents in mechanical energy, which is then used to drive an electrical generator $[1,2]$. The utilization of these renewable energy resources is crucial for the economic growth and the improvement of local living standards of many residents in remote areas without access to electricity $[3,4]$. Hydrokinetic power conversion has aroused great interest among the scientific community since when compared to other renewable energy sources, it is more predictable, reliable, stable, continuous and sustainable. Additionally, the hydrokinetic turbines allow the use of small low-head water resources; so that these turbines are environmentally friendly [3-5].

The performance of the hydrokinetic turbines depends on the blade or the rotor, shaft, gear box and the generator characteristics. However, the blade or the rotor is the most important component of the turbine system. To optimize the design of the blade, previous studies have reported the use of a variety of different approaches employing several geometries, angles of attack $(\alpha)$, number of blades, tip speed ratio (TSR) values and flow conditions using computational fluid dynamics (CFD) simulations to investigate the maximum power generated from the turbine and the maximum momentum extracted from the flow [4].

Traditionally, the blade of wind turbines and horizontalaxis hydrokinetic turbines is composed of twodimensional standard hydrofoil cross-sections; however, multi-element hydrofoil configurations or high-lift systems in the cross-section of turbine blades have been shown to improve the hydrodynamic characteristics of the blades, which can lead to an overall performance improvement of the turbines $[6,10]$. The multi-element configuration consists of several combinations of slats and flaps around a main hydrofoil [6 11]. The effectiveness of the multi-element hydrofoil configuration depends of the positions of the slat or the flap with respect to the main hydrofoil. The slot size between the main element and the high lift components (slat or flap) is defined in terms of the overhang and the gap. The overhang is the horizontal distance from the trailing edge of the upstream element to the leading edge of the downstream element. On the other hand, the gap is the minimum distance between the trailing edge of the upstream element and the leading edge of the downstream element. The position of the flap can be defined by three parameters, including the overlap, gap, and the slat and/or flap deflection angle $(\delta)[6,11,12,13]$.

Various studies focusing on the slat-airfoil configurations have been carried out. Results showed that the slat geometry, slat orientation with respect to the main airfoil, and other geometric parameters have an important effect on the performance of the hydrofoil $[7,8,9]$. In the case of a hydrofoil-flap arrangement for hydrokinetic turbines, there are few studies regarding hydrokinetic application. Aguilar et al. used a two-dimensional (2D) simulation to define an optimum orientation of a hydrofoil-flap arrangement for obtaining the maximum lift to drag ratio and avoiding the cavitation [12]. Nevertheless, from the authors' knowledge, three-dimensional (3D) CFD simulations and experimental researches have not been developed yet. Thus, the goal of this work is to perform a 
numerical investigation on the effect of a high-lift hydrofoil with a flap on a horizontal-axis hydrokinetic turbine using the 3D CFD technique, including the user-defined functions (UDF) of 6-degrees of freedom (6-DoF). For this purpose, the numerical results obtained are compared and discussed based on the experimental data available in the literature on the performance of a horizontal-axis hydrokinetic turbine whose blade has been designed with a hydrofoil traditional configuration.

\section{Numerical simulation}

Two models of rotor for the horizontal-axis hydrokinetic turbines shown in Fig. 1 were designed to study the behaviour of this kind of turbines with and without a highlift hydrofoil arrangement. The design of the rotors was carried out using the blade element momentum theory (BEM) [14]. For this purpose, the Eppler 420 foil geometry was selected to obtain the blades of the turbines. The rotors designed in this study were composed of three blades, with a diameter $(D)$ or radius $(R)$ equal to $1.58 \mathrm{~m}$ and $0.79 \mathrm{~m}$, respectively. The details of the utilized blade geometry are summarized in Table I, where $r$ refers to the location of each cross-section from the hub, $\beta$ is the blade twists angle and $C$ is the chord length of the hydrofoil. In the case of a rotor with a hydrofoil-flat arrangement, the optimum geometric parameters for the cross-section were obtained from 2D CFD simulation developed by Aguilar and co-workers [12]. Thus the design parameters of the high-lift system with a flap were an overlap of $8.52 \%$ of the chord length of the main element $C_{1}$, a gap of $2.825 \% C_{1}$ and a flap chord length $\left(C_{2}\right)$ of $42.47 \% C_{1}$. In turn, the optimized high-lift hydrofoil had $19.765^{\circ}$ and $-4^{\circ}$ as $\delta$ and $\alpha$, respectively [12].
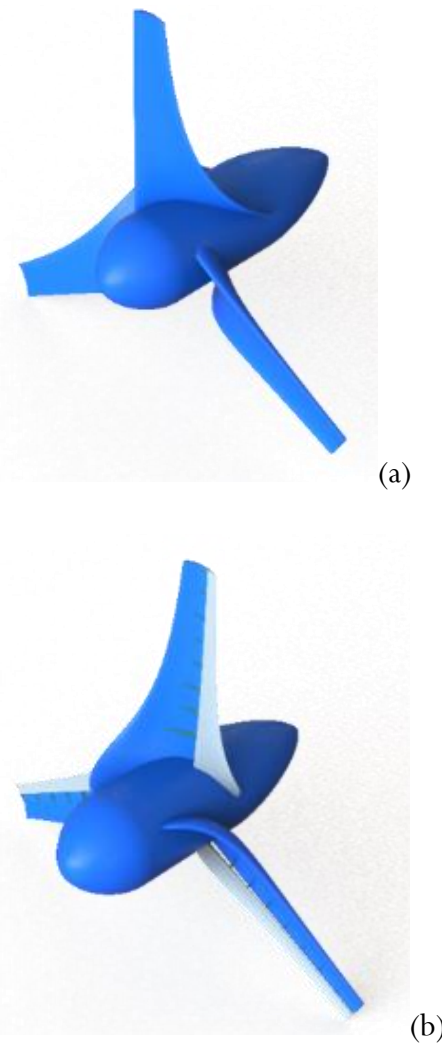

Fig. 1. Solid view of the rotors. a) Traditional rotor and b) high-lift rotor
Table I. Detailed blade characteristics

\begin{tabular}{|l|l|c|}
\hline$r / R$ & $\beta\left(^{\circ}\right)$ & $C / R$ \\
\hline 0.1 & 50.83 & 0.05949 \\
\hline 0.2 & 35.93 & 0.05063 \\
\hline 0.3 & 27.35 & 0.04050 \\
\hline 0.4 & 22.18 & 0.03291 \\
\hline 0.5 & 18.82 & 0.02785 \\
\hline 0.6 & 16.48 & 0.02405 \\
\hline 0.7 & 14.77 & 0.02152 \\
\hline 0.8 & 13.46 & 0.01899 \\
\hline 0.9 & 12.43 & 0.01772 \\
\hline 1.00 & 11.61 & 0.01646 \\
\hline
\end{tabular}

The behaviour of the rotors was studied using CFD. The methodology applied in all simulations was based on the unsteady Reynolds averaged Navier Stokes (URANS) equations and the shear stress transport (SST) $k-\omega$ turbulence model [15], which was performed by ANSYS Fluent software.

For this, a cylindrical volume was used as the computational domain, as represented in Fig. 2. The diameter of the entire computational domain was set as $4 \mathrm{D}$, the distance between the center point of the turbine and the inlet was fixed as 2D, and the distance between the center point and the outlet was set as 5D. The numerical domain was divided into two sub domains: i) an inner cylindrical rotatory zone containing the rotor and ii) a stationary zone. The cylindrical rotary zone was $2.5 \mathrm{D}$ and $\mathrm{D}$ in diameter and length, respectively. Between two domains, an interface condition was applied. A uniform velocity inlet boundary equal to $1.5 \mathrm{~m} / \mathrm{s}$ and a pressure outlet boundary were applied at the left and right surface of the domain, respectively. To improve the stability of the numerical simulations, symmetry boundary conditions was imposed at the side wall of the cylinder. No-slip boundary conditions were applied at the surface of the blade. An unstructured mesh with a viscous length of the mesh expressed as $y+<5$ was used around the wall of the blade.

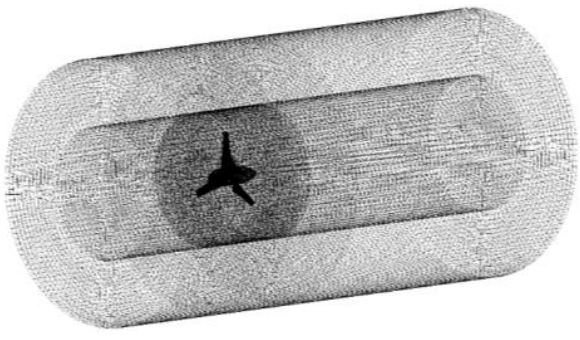

(a)

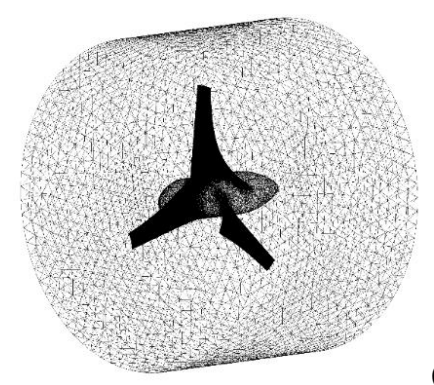

(b)

Fig. 2. a) Computational domain and b) inner cylindrical rotatory zone 
In this work, the turbine performance was primarily quantified by the power coefficient $\left(C_{P}\right)$, which was estimated by using Eq. (1).

$$
C_{P}=\frac{P}{\frac{1}{2} \rho V^{3} \pi R^{2}}
$$

where $P$ is the mechanical power output, $\rho$ is the fluid density, $V$ is the incoming velocity and $R$ is the radius of the turbine. Simulations were carried out for TSR ranging from 2.5 to 9 . TRS is a ratio of the turbine blade tangential speed compared to $V$. It was determined by using Eq. (2), where, $\boldsymbol{\omega}$ is the angular velocity.

$$
T S R=\frac{\omega R}{V}
$$

A sliding mesh and a 6-DOF function were utilized for modelling the rotation of the turbine and analysing the rigidbody dynamics. In contrast to a simulation with the TSR given, $\omega$ was not specified in the simulation. In this approach, the turbine blade rotated around its axis at a certain $\omega$ by balancing the hydrodynamic moment, the moment of inertia $(J)$ and the imposed counter moment on the turbine. This balance is represented by Eq. (3) $[16,17]$.

$$
J \frac{d \omega}{d t}=M_{F}-M_{A}
$$

where $d \omega / d t$ stands for the acceleration of the turbine, $M_{F}$ is the total hydrodynamic moment acting on the turbine blade and $M_{A}$ is the applied moment on the rotational axis for determining the power of the turbine. Hence, $\omega$ can be defined as expressed in Eq. (4).

$$
\omega=\int \frac{M_{F}-M_{A}}{J} d t
$$

Once the motion of the turbine rotor is determined, $\mathrm{P}$ can be obtained by using Eq. (5).

$$
P=M_{A} \omega
$$

To ensure the quality of the simulation, mesh and time-step independence tests were conducted before data analysis using the grid convergence index (GCI), which is a term coined by Roache based on Richardson extrapolation method. The GCI is a measure of how far the simulation results are from the asymptotic numerical result $[16,17,18]$.

The first step of the extrapolation is to determine the convergence coefficient or the order of accuracy of the numerical solution, which is notated as $p$, as shown in Eq. (6) $[17,18]$.

$$
p=\frac{\ln \left(\frac{f_{3}-f_{2}}{f_{2}-f_{1}}\right)}{\ln (r)}
$$

where $r$ is the ratio of refinement. The extrapolation of the exact value by Richardson extrapolation could be determined by Eq. (2). $f_{3}, f_{2}$, and $f_{1}$ are the control variable values for the convergence analysis of the coarse, medium and fine mesh or time-step, respectively. In this study, the control variable was the area under the curve defined by $C_{P}$ versus TSR [17,18].
The GCI for the fine solution $\left(G C I_{\text {fine }}\right)$ and the coarse solution $\left(G C I_{\text {coarse }}\right)$ are determined using Eq. (7) and (8), respectively. GCI provides an estimation of the amount of the discretization error in the finest grid solutions concerning the converged numerical solution. A low GCI implies that the solution does not considerably depend on the mesh or the time step sizes. The adequacy of the medium mesh or the time step was determined by comparing the GCI between the coarse and the medium grids (Eq. 7) and between the medium and the fine grids (Eq. 8) $[16,17,18]$.

$$
\begin{gathered}
G C I_{\text {coarse }}=\frac{F \cdot\left|\epsilon_{23}\right|}{r^{p}-1} \\
G C I_{\text {fine }}=\frac{F \cdot\left|\epsilon_{12}\right|}{r^{p}-1}
\end{gathered}
$$

where $\mathrm{F}$ is a safety factor of the method, with recommendations ranging from 1.0 to 3.0. The general recommendation is to use a $\mathrm{F}$ value equal to 1.25 when a minimum of three grids are used to verify the order of convergence, $p$ [18]. On the other hand, a conservative value of 3 is recommended when a major level of uncertainty of the estimated error exists and when the solution from two meshes is used to estimate the error. In turn, $\epsilon_{23}$ and $\epsilon_{12}$ are the relative error between subsequent solutions, which can be calculated as represented by Eq. (9) and Eq. (10) respectively[18].

$$
\begin{aligned}
& \epsilon_{23}=\frac{f_{3}-f_{2}}{f_{2}} \\
& \epsilon_{12}=\frac{f_{2}-f_{1}}{f_{1}}
\end{aligned}
$$

The extrapolation of the exact value by Richardson extrapolation could be determined as expressed by Eq. (11) $[17,18]$.

$$
f_{h=0}=f_{1}+\frac{f_{1}-f_{2}}{r^{p}-1}
$$

Finally, in order to guarantee that the mesh or the timestep sizes are in the asymptotic range of convergence, the index showed in Eq. (12) must be checked [18].

$$
I=\frac{G C I_{\text {Coarse }}}{r^{p} G C I_{\text {Fine }}} \cong 1
$$

where $I$ is the index of the asymptotic range of convergence. $I$ values approaching 1 mean that the simulation is within the asymptotic range. In this regard, for the discretization error due to both the space and the time discretization by using GCI approaches, three different mesh resolutions (fine mesh, medium mesh and coarse mesh with $3995127,4923646,5605713$ elements, respectively) and time-steps $(0.002 \mathrm{~s}, 0.001 \mathrm{~s}$ and 0.0005 $\mathrm{s})$ were used in this study. F was equal to 1.25 . In the case of the mesh independence test, $I$ was equal to 1.024 and 0.995 for the traditional and the high-lift configurations, respectively. On the other hand, $I$ was equal to 0.994 for the time-step independence study. The results demonstrated the independency of numerical results on the mesh and time-step sizes. Consequently, the medium mesh and the time-step equal to 0.001 were employed for all the successive simulations considered in this study since a good compromise in terms of results and computational time was achieved. Richardson extrapolation results for 
the mesh and the time-step independency were shown in Fig. 3 and 4, respectively.
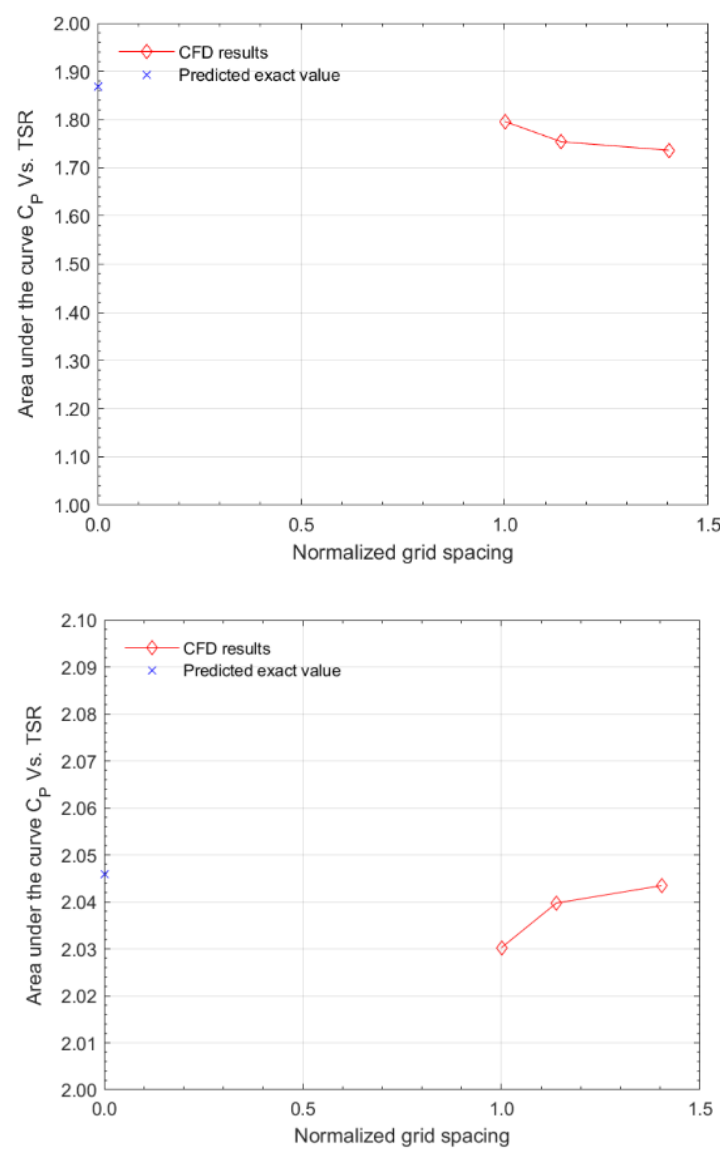

a)

Fig. 3. Mesh independence test results. a) Traditional rotor and b) high-lift rotor

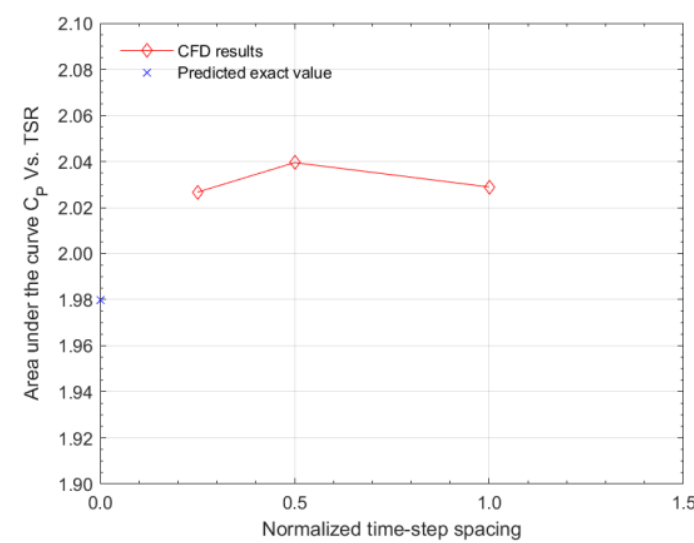

Fig. 4. Time-step independence test results.

\section{Results and discussion}

Fig. 5 shows the distribution of $C_{P}$ with different values of TSR using CFD approach and the experimental results reported in the literature. The numerical results for the traditional rotor were in good agreement with a previous study of this specific turbine model in terms of similar $C_{P}$ values, except that the $C_{P}$ peak was achieved at a different TSR value. The differences are due to the fact that the hydrofoil used in the study of Tian and co-workers was the FF-77-W airfoil type [19].

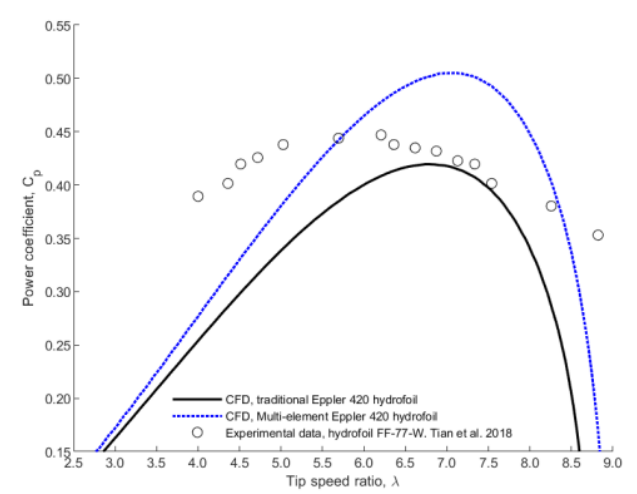

Fig. 5. Variation of the power coefficient $\left(C_{P}\right)$ vs. the tip speed ratio $(T S R)$

The maximum $C_{P}$ values obtained were 0.5050 and 0.419 for the high-lift hydrofoil and the traditional configurations, respectively.

\section{Conclusion}

Using URANS solver and 6-DOF function, 3D CFD studies have been conducted to predict the performance of two rotors, defined by the $C_{P}$ achieved at different TSR values. The results demonstrated that the rotor with a highlift hydrofoil enhanced the $C_{P}$ compared to the rotor with a single-element hydrofoil arrangement. From the 3D CFD analyses for the high-lift hydrofoil, the maximum efficiency found was 0.5050 , while a maximum efficiency of 0.419 was obtained with the traditional hydrofoil configuration. The validation showed that the CFD results concerning the traditional hydrofoil configuration were in good agreement with the empirical data examined. In this study, GCI was a good option for estimating the appropriate sizes of the time-step and the mesh.

\section{Acknowledgement}

The authors gratefully acknowledge the financial support provided by the Colombia Scientific Program within the framework of the call Ecosistema Científico (Contract No. FP44842-218-2018).

\section{References}

[1] Maldar, N. R., Ng, C. Y., and Oguz, E. A review of the optimization studies for Savonius turbine considering hydrokinetic applications. Energy Conversion and Management (2020). Vol. 226, 113495.

[2] Lust, E. E., Bailin, B. H., and Flack, K. A. Performance characteristics of a cross-flow hydrokinetic turbine in current only and current and wave conditions. Ocean Engineering (2021). Vol. 219, 108362.

[3] John, B., and Varghese, J. Sizing and techno-economic analysis of hydrokinetic turbine based standalone hybrid energy systems. Energy (2021). Vol. 221, 119717.

[4] Yuce, M. I., and Muratoglu, A. Hydrokinetic energy conversion systems: A technology status review. Renewable and Sustainable Energy Reviews (2015). Vol. 43, pp. 72-82. 
[5] Kirke, B. Hydrokinetic turbines for moderate sized rivers. Energy for Sustainable Development (2020). Vol. 58, pp. 182-195.

[6] Narsipur A., Pomeroy B., and Selig M. CFD Analysis of multielement airfoil for wind turbines. 30th AIAA Applied Aerodynamics Conference (2012). pp. 2781.

[7] Yavuza, T., Kilkis, B., Koc, E., and Erol, O. Flow and performance characteristics of a double-blade hydrofoil. In Advanced Materials Research (2012). Vol. 433, pp. 7218-7222.

[8] Yavuz, T., and Koç, E. Performance analysis of double blade airfoil for hydrokinetic turbine applications. Energy conversion and management (2012). Vol. 63, pp. 95-100.

[9] Yavuz, T., Koç, E., Kılkış, B., Erol, Ö., Balas, C., and Aydemir, T. Performance analysis of the airfoil-slat arrangements for hydro and wind turbine applications. Renewable energy (2015). Vol. 74, pp. 414-421.

[10] Hashemi, S. M., Moghimi, M., and Derakhshan, S. Experimental and numerical study of a flapping-blade vertical-axis hydrokinetic turbine under free surface deformation and blockage effects. International Journal of Environmental Science and Technology (2020). Vol. 17(8), pp. 3633-3650.

[11] Ragheb, A., and Selig, M. Multi-element airfoil configurations for wind turbines. In 29th AIAA Applied Aerodynamics Conference (2011). pp. 3971.

[12] Aguilar, J., Rubio-Clemente, A., Velasquez, L., and Chica, E. Design and Optimization of a Multi-Element Hydrofoil for a Horizontal-Axis Hydrokinetic Turbine. Energies (2019). Vol. 12(24), pp. 4679.
[13] Jeong, S., Murayama, M., and Yamamoto, K. Efficient optimization design method using kriging model. Journal of aircraft (2005). Vol. 42(2), pp. 413-420.

[14] Manwell, J. F., McGowan, J.G., and Rogers, A.L. Aerodynamics of Wind Turbines. In: J. F Manwell, JG McGowan and AL Rogers (eds) Wind energy explained: theory, design and application. 2th ed. UK: John Wiley \& Sons 2009, cap. 3, pp. 91-155.

[15] Menter, F.R. Two-equation eddy-viscosity turbulence models for engineering applications. AIAA journal (1994). Vol. 32(8), pp. 1598-1605.

[16] Prakoso, A. P., Adanta, D., and Irwansyah, R. Approach for a breastshot waterwheel numerical simulation methodology using six degrees of freedom. Energy Reports (2020). Vol. 6, pp. 611-616.

[17] Prakoso, A. P., Siswantara, A. I., and Adanta, D. Comparison between 6-DOF UDF and moving mesh approaches in CFD methods for predicting cross-flow pico-hydro turbine performance. CFD Letters (2019). Vol. 11(6), 86-96.

[18] Roache, P. J. Perspective: a method for uniform reporting of grid refinement studies. Journal of Fluids Engineering (1994). Vol. 116, pp. 405-413.

[19] Tian, W., Mao, Z., and Ding, H. Design, test and numerical simulation of a low-speed horizontal axis hydrokinetic turbine. International Journal of Naval Architecture and Ocean Engineering (2018). Vol. 10(6), pp. 782-793 